\title{
TerraSAR-X Based Monitoring of Mangrove Reforestation in Senegal: A Feasibility Study
}

\author{
Keith PETERSON ${ }^{1,2}$ and Felicitas VON PONCET ${ }^{1}$ \\ ${ }^{1}$ Astrium GEO-Information Services, Immenstaad/Germany · keith.peterson@astrium.eads.net \\ ${ }^{2}$ UNIGIS, Paris Lodron University Salzburg/Austria
}

This contribution was double-blind reviewed as extended abstract.

\section{Introduction}

Mangrove ecosystems are rapidly disappearing worldwide at a rate of $1-2 \%$ annually due to a combination of climate change and increased anthropogenic pressure and if the current trend continues unabated, mangrove forests may vanish completely within the next 100 years (DUKE et al. 2007). In an extensive review of the status of the world's mangroves between 1980 and 2005, the FAO (2007) calculated a decrease in mangrove forest area of approximately $20 \%$. The nation of Senegal, in cooperation with Oceanium (a local NGO) and the Livelihoods Fund (a carbon investment fund), has been supporting the replanting of mangrove forests around vulnerable coastlines in the Sine Saloum and Casamance estuaries within the framework of a Clean Development Mechanism (CDM). The difficulty that the Livelihood Fund faces is the continual monitoring of these plantations, allowing investors to track the value of their investment as the forest grows. Presently, solely ground based sampling schemes are used to collect the necessary information which is both expensive and limited by accessibility. A remote sensing based approach, using satellite imagery, can potentially provide wall-to-wall coverage of the plantation areas and increase the level of efficiency in which the project is evaluated by optimizing the amount of field data needed for an accurate assessment of the plantations. To-date, the monitoring of young mangrove reforestation efforts has not been performed with remote sensing based techniques (FIELD 1999). The main objective of the current study is to consider the potential for using TerraSAR-X for the routine monitoring of young mangrove plantations, between 1 and 5 years old, and to determine if tree density (number of tree per hectare) can be accurately derived from the data. In addition, the acquisition mode of TerraSAR-X was examined to determine the optimum data parameters. The study investigated 3 groups of plantations, established between 2008 and 2012, in the Casamance estuary near the communities of Thiobon, Cap Skiring and Ziguinchor in south western Senegal, located between $12^{\circ} 15^{\prime}$ and $13^{\circ} 06^{\prime}$ North and $16^{\circ} 15^{\prime}$ and $16^{\circ} 48^{\prime}$ 'West.

\section{Input Data and Data Processing}

In order to evaluate the potential of TerraSAR-X, images were acquired in three different acquisition modes ranging in resolution between 1-3 meters: High Resolution SpotLight, SpotLight and StripMap. For all modes, repeat pass acquisitions at 11 day time intervals 
were acquired to study temporal backscatter variation and the influence of water level changes. The images were acquired during the beginning of the dry season and parallel to the 2011 and 2012 field campaigns (24.11.2011 - 27.12.2011, and 24.10.2012 24.12.2012). Field samples were collected within the plantations using GPS. The field team collected detailed information on planted tree density, height and diameter within a $10 \mathrm{~m}$ radius $\left(314 \mathrm{~m}^{2}\right)$ plot area. In addition, photographs were taken and the photo numbers were recorded for each field sample. The survey team also provided a limited number of field delineated "growth quality" polygons within the plantations. The growth quality attribute refers to the quality of tree growth (i.e. biophysical parameters) and not to the number or density of trees. TerraSAR-X SSC data were used to process the interferometric coherence (complex phase correlation) between repeat passes, whereas calibrated backscatter intensity was derived from EEC RE (radiometrically enhanced), as provided by the TerraSAR-X Ground segment, providing a noise correction particularly important for surfaces with backscatter close to noise floor (NESZ).

\section{Methods for the Estimation of Tree Density}

In order to investigate the relationship between backscatter and plant characteristics, a priori and field information, such as planting year, plant density, height and DBH were used to separate data into different collections and analyse their behaviour with respect to specific criteria. Additionally, the influence of tide level was indirectly assessed by a) comparing scenes of the same area acquired at different dates and b) comparing samples located in test sites acquired with high water level (Ziguinchor) to others showing lower water level. Tide level information available for 2 gauges along the Casamance River and some water level measurements within the plantations support the interpretation. Further analysis investigated if interferometric coherence is related to plant parameters and waterlevel changes. The usefulness of X-band InSAR observations for monitoring water level changes in aquatic environments with emergent vegetation was recently examined by HoNG et al. (2010). The above described investigations were based on mean backscatter and coherence extracted for the sample plot (a $10 \mathrm{~m}$ buffer was applied to each sample plot GPS point to simulate the actual plot size).

\section{Results}

The high spatial and temporal variation of the water level within the plantation areas is a major challenge for the data analysis. Water level measurements at the time of image acquisition are available for 6 sites with varying plant size and density which supports the interpretation. The presence of water significantly affects the SAR backscatter. The most important scattering mechanisms for monitoring young mangrove plantations are doublebounce signals between the water and the vertical tree structures. In this case, the backscatter intensity is governed by plant density, provided that a relevant proportion of the vertical plant structure emerges from the water. In order to accurately establish a relationship between the field-measured tree density and TSX backscatter, zonal statistics computed for the field samples had to be filtered and separated into groups representing different operating conditions. The samples are grouped into 3 water-level-based conditions present at the time of acquisition: 1) submerged, where only a small part of plant structure 
is exposed, resulting in a very low sensitivity of backscatter to plant parameters, characterized by low backscatter and coherence; 2) exposed, where the dielectric properties and surface roughness of the soil surface has a strong influence on backscatter, resulting in high backscatter and coherence if $T_{1}$ and $T_{2}$ are not flooded; and 3) flooded (low to medium water level), proved optimal to retrieve information on plant parameters, as the water surface provides the ideal background to measure small plant structures. The relationship improves with increasing plant height, as a larger proportion of the plant is above water, thus offering a sufficient backscattering surface. A higher coherence level was observed for larger plants, given $T_{1}$ and $T_{2}$ are both flooded. Since the 2012 field campaign focused on the assessment of freshly planted sites, most of the samples stem from very small saplings, resulting in submerged conditions under the given high tide level for many of the investigated acquisitions. Thus, field plots with $<40 \mathrm{~cm}$ tree height have been excluded from the sample set, leaving samples which were split into equal sized datasets for model development and validation by random selection. Fig. 2 (left) shows the relationship of backscatter to plant density for group 3 (representing favourable conditions) with an $\mathrm{R}^{2}$ of 0.68. Fig. 2 (right) shows measured versus predicted values with an RMSE of 1455 plants/ha, representing $12 \%$ of the observed range of plant density.
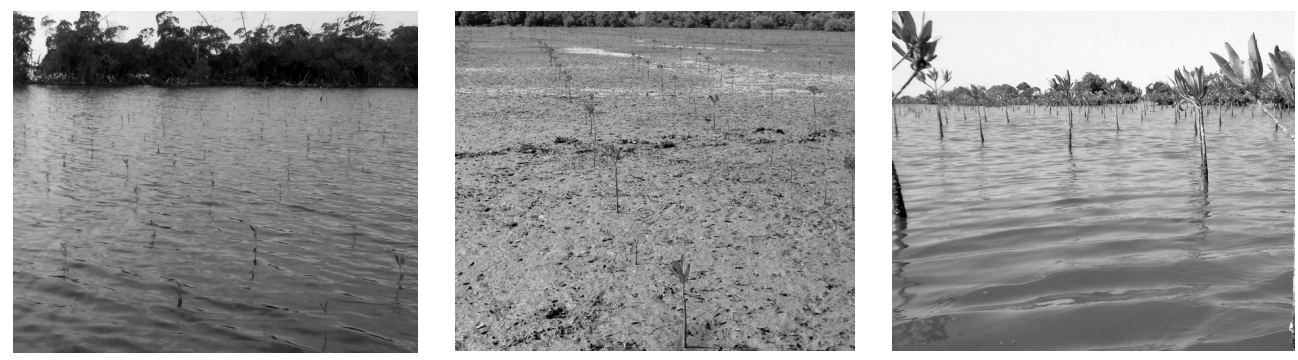

Fig. 1: Field photos representing the varying operating conditions: high tide, low tide and low-medium water level (from left to right)
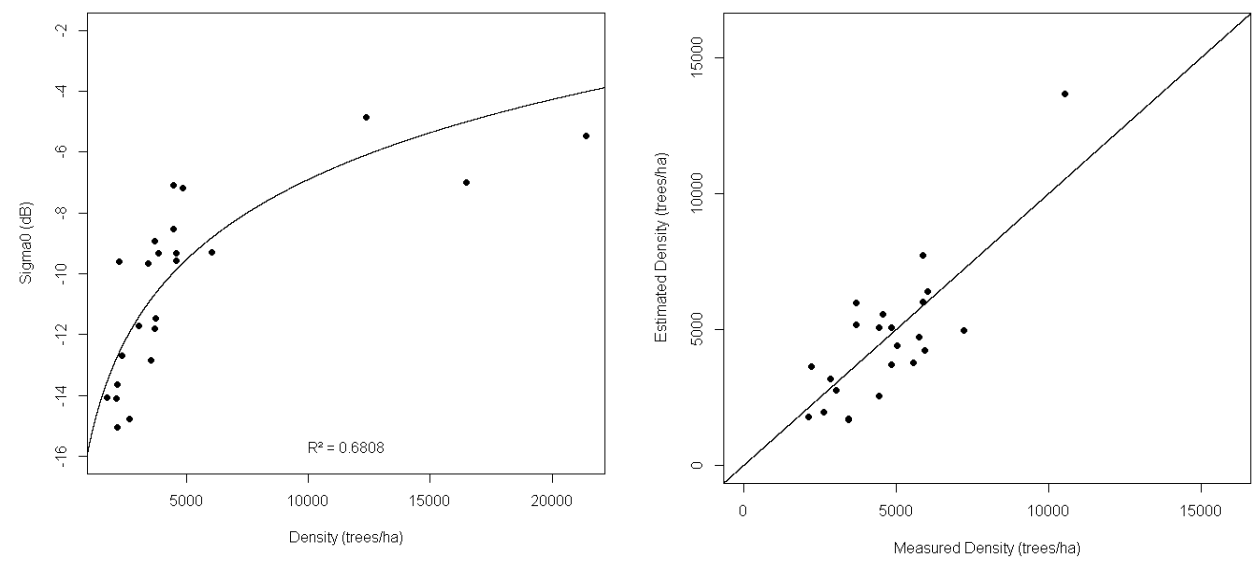

Fig. 2: Density vs. backscatter for the modelling points $\left(\mathrm{R}^{2}=0.68\right)$ in comparison to control points (left); estimated vs. field measurements with an RMSE of $12 \%$ (right) 


\section{Conclusion and Outlook}

TerraSAR-X StripMap resolution was proven sufficient for mapping within plantation variations. This is important for the future development of an efficient plantation monitoring strategy, as the StripMap mode provides optimal coverage $(30 \times 50 \mathrm{~km})$ and spatial resolution $(3 \mathrm{~m})$, thus minimizing costs for the end user. The sensors short wavelength $(3 \mathrm{~cm})$ is well suited for the detection of small plant structures despite varying operating conditions.

The results demonstrate that, given the appropriate operating conditions, a relationship exists between TerraSAR-X backscatter and tree density in young mangrove plantations up to 4 years in age. Radar backscatter explains between $68 \%$ (2012 results) and $90 \%$ (2011 results) of the plant density variance measured in the field depending on acquisition conditions and plant age. The coefficient of determination decreased considerably between 2011 and 2012. This is the result of the concentration of 2012 field sampling on younger plantations, more influenced by background surface conditions than more developed plants. However, provided that 2012 sampling is closer to operational conditions, the results of the error statistics for the validation data set (12\% RMSE) exhibit a very useful result for the control of mangrove plant growth. The higher uncertainties for the groups of non-optimal conditions can be compensated by more field samples in a stratified sampling approach, combining remote sensing and field survey data. In the next steps, objective criteria derived from amplitude and phase information will be defined to assign homogeneous plantation areas to one of the 3 groups. The efficiency of the stratification will depend on the areal proportion of plantations showing a strong correlation to plant densities.

While this study has provided a sound analysis of the potential of high resolution X-band radar for the monitoring of young mangrove plantations, a substantial amount of work remains in order to refine the results for the development of an efficient, transferable methodology for the quantification of tree density variation within all the plantations associated with the project.

\section{References}

Duke, N. C., Meynecke, J.-O., Dittmann, S., Ellison, A. M., Anger, K., Berger, U., Cannicci, S., Diele, K., Ewel, K. C., Field, C. D., Koedam, N., Lee, S. Y., MarchAnd, C., Nordhaus, I. \& DajdouH-Guebas, F. (2007), A World Without Mangroves? Science, 317,3 .

FAO (2007), The world's mangroves 1980-2005. FAO Forestry, 153, 89.

FIELD, C. D. (1999), Mangrove rehabilitation: choice and necessity. Hydrobiologia, 413, 5. HonG, S.-H., WDOwINSKI, S. \& KIM, S.-W. (2010), Evaluation of TerraSAR-X Observations for Wetland InSAR Application. IEEE Transactions on Geoscience and Remote Sensing, 48 (2), 10. 